\title{
AN ASYMPTOTIC ANALYSIS OF THE PERIOD-DOUBLING SECONDARY BIFURCATION IN A FILM/SUBSTRATE BILAYER*
}

\author{
Y. B. $\mathrm{FU}^{\dagger}$ AND Z. X. CAI ${ }^{\ddagger}$
}

\begin{abstract}
It has previously been observed experimentally and simulated numerically that when a thin film bonded to a much softer substrate is subjected to a uni-axial compression parallel to the interface, the initial buckled pattern will suffer a secondary bifurcation that doubles the period of the original pattern when the compressive strain reaches a critical value. This perioddoubling phenomenon is analyzed in this paper using an asymptotically self-consistent approach based on the exact theory of nonlinear elasticity. The predicted critical strain based on a fourterm expansion shows good agreement with that obtained using fully numerical simulations, and it is demonstrated that four is the minimum number of terms that should be included in order to give realistic predications. Although our illustrative calculations are conducted for neo-Hookean materials, the proposed approach can deal with any material models and can be extended to higher orders.
\end{abstract}

Key words. bilayer, period-doubling, nonlinear elasticity, tunable patterns

AMS subject classification. 74B20

DOI. $10.1137 / 15 \mathrm{M} 1027103$

1. Introduction. Strain-induced pattern formation, traditionally referred to as buckling, which is usually undesirable in engineering applications, is now recognized to have applications at the micrometer and submicrometer scales ranging from cell patterning, optical gratings, and creation of surfaces with desired wetting and adhesion properties, to metrology of ultrathin film properties; see Bowden et al. [2, 3], Li et al. [12], and the references therein. As a result, microstructures forming on a hard thin film that is bonded to a soft substrate have received a lot of attention in recent years. Of particular interest is the period-doubling secondary bifurcation that has been observed experimentally by Pocivavsek et al. [15], Brau et al. [4], and Sun et al. [16], and numerically simulated by Sun et al. [16], Cao and Hutchinson [8], and Budday, Kuhl, and Hutchinson [5]. This phenomenon has also been analyzed theoretically based on various approximate theories by Brau et al. [4], Zhao et al. [17], and Zhuo and Zhang [18]. Although successful at providing important first insight into the period-doubling phenomenon, justification of the various assumptions behind these approximate theories can be very subtle $[7,1]$, and their self-consistency in the asymptotic sense remains unclear. Thus, it is not entirely unexpected that the analysis of Brau et al. [4] predicts nonexistence of period-doubling if the substrate is incompressible, which contradicts the results from fully numerical simulations given by Cao and Hutchinson [8] and Zhao et al. [17]. In this paper we examine the period-doubling phenomenon based on the exact theory of nonlinear elasticity without making any approximating assumptions about the interfacial conditions or the nonlinear behavior of the bilayer structure. Our only approximation is that the primary buckled state can

*Received by the editors June 22, 2015; accepted for publication (in revised form) August 25, 2015; published electronically November 3, 2015. This work was supported by the National Natural Science Foundation of China (grants 11372212 and 11172200), and by the National Basic Research Program of China (grant 2013CB035402).

http://www.siam.org/journals/siap/75-6/M102710.html

${ }^{\dagger}$ Department of Mechanics, Tianjin University, Tianjin, China, and Department of Mathematics, Keele University, Staffordshire ST5 5BG, UK (y.fu@keele.ac.uk).

${ }^{\ddagger}$ Department of Mechanics, Tianjin University, Tianjin, China (zxcai@tju.edu.cn). 
be described by a weakly nonlinear analysis (Cai and $\mathrm{Fu}[6]$, hereafter referred to as $\mathrm{CF}$ ), but we take into account the fundamental buckling mode and its harmonics up to and including at least the third order and justify the assumption asymptotically a posteriori. We use the dynamical systems approach, as in [4]; this contrasts with the energy approach favored in the other theoretical studies.

The rest of this paper is organized as follows. Our point of departure is our previous paper $\mathrm{CF}$, where an exact weakly nonlinear postbuckling analysis was conducted to determine the critical ratio of shear moduli below which the primary bifurcation is subcritical (and so is sensitive to imperfections; see also Hutchinson [11]). Although the dynamical systems theory view of period-doubling is now classical and can be found in, for instance, Nayfey and Mook [13], it would be instructive to summarize its methodology through a simple example before we apply it to the incremental equations relevant to our current problem. This is done in the next section, which is then followed in section 3 by a summary of the incremental equations derived from nonlinear elasticity theory. We look for an asymptotic solution for the threshold stretch associated with period-doubling in terms of a small parameter that characterizes the deviation of the stretch from its critical value corresponding to the primary bifurcation. The first term in the asymptotic expansion is the buckling stretch at which the mode with twice the period of the primary buckling mode can bifurcate from the uniformly stretched state. The next three terms are derived in sections 4 and 5, with illustrative numerical results presented in section 6 and compared with existing results from experimental studies and numerical simulations. The paper is concluded with a summary and a brief discussion of the effects of higher-order terms.

2. Formulation and solution strategy. We consider an elastic body $B$ which consists of a finite-thickness layer of hard material bonded to a (semi-infinite) substrate of soft material. We assume that $B$ possesses an initial unstressed configuration $B_{0}$. A purely homogeneous static deformation is imposed upon $B_{0}$ to produce a finitely stressed equilibrium configuration denoted by $B_{e}$. In our illustrative calculations this homogeneous deformation is assumed to be a uniaxial compression parallel to the interface. We do not consider a prestretch in the substrate before it is bonded to the layer, but our formulation can easily be modified to take into account this extra feature. When the homogeneous deformation reaches a critical level, the bilayer may prefer to buckle into an inhomogeneous configuration, denoted by $B_{t}$, in which the extra deformation is sinusoidal in the direction parallel to the interface and decays to zero exponentially away from the interface into the substrate. The position vectors of a representative material particle relative to a common coordinate system are denoted by $\boldsymbol{X}, \boldsymbol{x}$, and $\tilde{\boldsymbol{x}}$, with components $X_{A}, x_{i}\left(X_{A}\right)$, and $\tilde{x}_{i}\left(X_{A}\right)$ in $B_{0}, B_{e}$, and $B_{t}$, respectively. In terms of $\boldsymbol{x}$, the bilayer structure is defined by

$$
0 \leq x_{2} \leq h \text { for the layer and }-\infty<x_{2} \leq 0 \text { for the substrate, }
$$

with the other two coordinates both varying between $-\infty$ and $\infty$. The constant $h$ in the above equation thus denotes the layer thickness in $B_{e}$.

We write

$$
\tilde{\boldsymbol{x}}(\boldsymbol{X})=\boldsymbol{x}(\boldsymbol{X})+\epsilon \boldsymbol{u}(\boldsymbol{x}),
$$

where $\epsilon \boldsymbol{u}$ denotes the displacement vector field associated with the deformation from $B_{e}$ to $B_{t}$, and $\epsilon$ is a small positive parameter characterizing the amplitude of this deformation. Throughout this paper, the incremental deformations are assumed to be plane-strain, so that here $u_{3}$ is zero and $u_{1}$ and $u_{2}$ are independent of $x_{3}$. 
Denote by $\lambda$ the stretch in the above-mentioned uniaxial compression, and by $\lambda_{\mathrm{cr}}$ its critical value at which bifurcation into a sinusoidal pattern first becomes possible. Denote the associated critical mode number by $k_{\mathrm{cr}}$, and write

$$
\lambda=\lambda_{\mathrm{cr}}-\epsilon^{2},
$$

where the small parameter $\epsilon$ is now given a definite meaning, and by choosing the sign in front of $\epsilon^{2}$ to be negative we have implicitly assumed that the bifurcation is supercritical; see CF. Note that in the bifurcation condition the mode number $k$ and film thickness $h$ appear through the product $k h$, and so if the critical stretch $\lambda_{\text {cr }}$ is attained at $k h=k_{\mathrm{cr}}^{(0)}$, say, then we have $k_{\mathrm{cr}}=k_{\mathrm{cr}}^{(0)} / h$. Without loss of generality, we may choose $1 / k_{\mathrm{cr}}$ as the unit for $x_{i}$ and $u_{i}$ so that the mode number becomes unity, and in the subsequent analysis the (nondimensional) film thickness $h$ takes the value $k_{\mathrm{cr}}^{(0)}$.

It was shown in $\mathrm{CF}$ that the postbuckling solution may be written in the form

$$
\epsilon \boldsymbol{u}(\boldsymbol{x})=\epsilon \boldsymbol{u}^{(1)}(\boldsymbol{x})+\epsilon^{2} \boldsymbol{u}^{(2)}(\boldsymbol{x})+\epsilon^{3} \boldsymbol{u}^{(3)}(\boldsymbol{x})+O\left(\epsilon^{4}\right),
$$

with

$$
\begin{aligned}
u_{1}^{(1)}= & A H^{\prime}\left(x_{2}\right) \mathrm{e}^{\mathrm{i} x_{1}}+\text { c.c. }, \quad u_{2}^{(1)}=-\mathrm{i} A H\left(x_{2}\right) \mathrm{e}^{\mathrm{i} x_{1}}+\text { c.c. } \\
& \boldsymbol{u}^{(2)}=\boldsymbol{U}^{(2,0)}\left(x_{2}\right)+\boldsymbol{U}^{(2,2)}\left(x_{2}\right) \mathrm{e}^{\mathrm{i} 2 x_{1}}+\text { c.c. }
\end{aligned}
$$

where for the case when both the layer and substrate are made of a neo-Hookean material, CF gave explicit expressions for the functions $H, U^{(2,0)}, U^{(2,2)}$, and the amplitude $A$; in the above expressions, c.c. denotes the complex conjugate of the term immediately preceding it. More precisely, due to the translational invariance of the configuration $B_{e}$ with respect to $x_{1}$, it is $|A|^{2}$ that is determined. Thus, once $|A|^{2}$ is known for each specified $\epsilon, A$ can be taken to be purely real or purely imaginary without loss of generality. To compare with existing studies, we shall take $A$ to be purely imaginary so that the vertical displacement $u_{2}^{(1)}$ is proportional to $\cos x_{1}$. We observe that the amplitude of the second term on the right-hand side of (2.2) is proportional to $\sqrt{\lambda_{\mathrm{cr}}-\lambda}$, which is typical of pitchfork bifurcations.

It turns out that for the purpose of predicting the threshold stretch for the secondary period-doubling bifurcation, the two-term solution given by $\mathrm{CF}$ is not enough, and we need to include terms at least up to order $O\left(\epsilon^{3}\right)$ in (2.4). Closed-form solutions for $\boldsymbol{u}^{(n)}(\boldsymbol{x})(n \geq 3)$ are no longer possible, but fortunately these higher-order solutions can easily be determined with the aid of Mathematica [19]. A code has been written that can determine the postbuckling solution to any order; our calculations are only limited by the computing time and gradual loss of accuracy as we proceed to higher orders. To sketch the solution structure, we note that the $n$th term takes the form

$$
\boldsymbol{u}^{(n)}(\boldsymbol{x})=\boldsymbol{U}^{(n, 0)}\left(x_{2}\right)+\sum_{k=1}^{n} \boldsymbol{U}^{(n, k)}\left(x_{2}\right) \mathrm{e}^{\mathrm{i} k x_{1}}+\text { c.c. },
$$

where the vector functions $\boldsymbol{U}^{(n, 0)}, \boldsymbol{U}^{(n, 1)}, \ldots, \boldsymbol{U}^{(n, n)}$ are determined using a standard perturbation procedure. It is observed that the problem for $\boldsymbol{U}^{(n, 1)}$ can be determined only if a solvability condition is imposed; this solvability condition would yield an 
algebraic equation for the amplitude of $\boldsymbol{U}^{(n-2,1)}$. Thus, to determine $\boldsymbol{u}^{(3)}(\boldsymbol{x})$ completely, we need to solve the problem for $\boldsymbol{u}^{(4)}(\boldsymbol{x})$ completely and the problem for $\boldsymbol{u}^{(5)}(\boldsymbol{x})$ partially in the sense that we only need to obtain the solvability condition on the problem for $\boldsymbol{U}^{(5,1)}$ in order to fix the amplitude of $\boldsymbol{U}^{(3,1)}$. Finally, we remark that although, according to the above procedure, the solution for $\boldsymbol{u}^{(2)}(\boldsymbol{x})$ should also contain the terms $\boldsymbol{U}^{(2,1)}\left(x_{2}\right) \mathrm{e}^{\mathrm{i} x_{1}}+$ c.c., it is found from the solvability condition on $\boldsymbol{U}^{(4,1)}\left(x_{2}\right)$ that these terms are in fact zero and are hence absent from (2.6).

To complete the description of the primary buckling solution, we assume that the pressure in $B_{t}$ associated with the constraint of incompressibility has the expansion

$$
p=\bar{p}+\left(\epsilon P_{1}+\epsilon^{2} P_{2}+\epsilon^{3} P_{3}+\cdots\right),
$$

where the first term is the pressure in $B_{e}$ and the second part represents the expansion of the incremental pressure, the $P_{n}$ having a similar representation to the $\boldsymbol{u}^{(n)}$ in (2.7).

Based on the existing experimental and numerical studies, we expect that as $\epsilon$ is increased to a certain threshold value, the above postbuckling solution will suffer a secondary period-doubling bifurcation. A subharmonic mode with mode number $1 / 2$ will first appear and then grow at the expense of the primary buckling mode defined above. Our interest in the present paper is in the determination of the threshold value of stretch at which the subharmonic mode first appears. This aim will be achieved with the aid of the dynamical systems theory approach. Before proceeding to detailed derivations and calculations, we first summarize this methodology by deriving the counterpart of our results for the following Mathieu equation:

$$
\ddot{w}+\left(\omega^{2}+2 \epsilon a_{1} \cos x_{1}+2 a_{0} \epsilon^{2}+2 a_{2} \epsilon^{2} \cos 2 x_{1}\right) w=0,
$$

where $w$ is a scalar function of the spatial variable $x_{1}$, a superimposed dot denotes differentiation with respect to $x_{1}$, the small parameter $\epsilon$ has the same meaning as in (2.3), and $a_{0}, a_{1}, a_{2}$ are known constants. The terms $2 \epsilon a_{1} \cos x_{1}$ and $\epsilon^{2}\left(2 a_{0}+\right.$ $2 a_{2} \cos 2 x_{1}$ ) would mimic the leading- and second-order terms in the weakly nonlinear solution given by (2.4). The $\omega$ would play the role of the principal stretch $\lambda$. As is the case in all linear buckling analysis, (2.9) always has the trivial solution, but we are looking for the threshold value of $\omega$ at which a nontrivial periodic solution with period $4 \pi$ can exist.

We thus write

$$
\omega^{2}=\frac{1}{4}+\epsilon \omega_{1}+\epsilon^{2} \omega_{2}+\cdots
$$

and look for a solution of the form

$$
w=w_{0}(x)+\epsilon w_{1}(x)+\epsilon^{2} w_{2}(x)+\cdots .
$$

On substituting (2.10) and (2.11) into (2.9) and equating the coefficients of like powers of $\epsilon$, we obtain

$$
\begin{gathered}
\ddot{w}_{0}+\frac{1}{4} w_{0}=0, \\
\ddot{w}_{1}+\frac{1}{4} w_{1}=-\left(\omega_{1}+2 a_{1} \cos x_{1}\right) w_{0}, \\
\ddot{w}_{2}+\frac{1}{4} w_{2}=-\left(\omega_{1}+2 a_{1} \cos x_{1}\right) w_{1}-\left(\omega_{2}+2 a_{0}+2 a_{2} \cos 2 x_{1}\right) w_{0} .
\end{gathered}
$$

Copyright $@$ by SIAM. Unauthorized reproduction of this article is prohibited. 
As anticipated, the leading-order problem (2.12) has the general solution $w_{0}=c_{1} \cos \frac{1}{2} x_{1}$ $+c_{2} \sin \frac{1}{2} x_{2}$, where $c_{1}$ and $c_{2}$ are constants. The right-hand side of (2.13) may then be written as

$$
-\left(\omega_{1}+a_{1}\right) c_{1} \cos \frac{1}{2} x_{1}-\left(\omega_{1}-a_{1}\right) c_{2} \sin \frac{1}{2} x_{1}-a_{1} c_{1} \cos \frac{3}{2} x_{1}-a_{1} c_{2} \sin \frac{3}{2} x_{1} .
$$

To eliminate secular/resonant terms, or equivalently to ensure that $w_{1}$ is still periodic, we must impose the conditions

$$
\left(\omega_{1}+a_{1}\right) c_{1}=0, \quad\left(\omega_{1}-a_{1}\right) c_{2}=0 .
$$

Two cases then follow. Either $\omega_{1}=-a_{1}, c_{2}=0$, or $\omega_{1}=a_{1}, c_{1}=0$. Taking $\omega_{1}=-a_{1}, c_{2}=0$, we obtain

$$
w_{0}=c_{1} \cos \frac{1}{2} x_{1}, \quad w_{1}=\frac{1}{2} a_{1} c_{1} \cos \frac{3}{2} x_{1} .
$$

At the next order, elimination of secular terms from the solution for $w_{2}$ then yields

$$
\omega_{2}=-2 a_{0}-\frac{1}{2} a_{1}^{2}
$$

In a similar fashion, the other choice $c_{1}=0$ would give the same value for $\omega_{2}$, although the corresponding $\omega_{1}$ is different. Thus, for this simple problem, the threshold value of $\omega$ at which a period-doubling solution can emerge is given by

$$
\omega=\frac{1}{4} \mp a_{1} \epsilon-\left(2 a_{0}+\frac{1}{2} a_{1}^{2}\right) \epsilon^{2}+O\left(\epsilon^{3}\right),
$$

with the $\mp$ signs corresponding to solutions that are in phase or out of phase, respectively, with the primary solution.

3. Incremental problem for the secondary bifurcation. We now superimpose on the configuration $B_{t}$ another infinitesimally small incremental deformation and denote the resulting configuration by $B_{t}^{*}$. The incremental equations are well documented; see, for instance, Ogden [14] or Fu and Ogden [10]. They are given by

$$
\begin{gathered}
v_{i, i}=0, \quad \chi_{i j, j}=0, \\
\chi_{i j}=\mathcal{A}_{j i l k} v_{k, l}+p v_{j, i}-p^{*} \delta_{j i},
\end{gathered}
$$

where $\chi$ is the incremental stress tensor, $\boldsymbol{v}$ is the incremental displacement from $B_{t}$ to $B_{t}^{*}$, and $p$ and $p^{*}$ are the incremental pressure fields (associated with the constraint of incompressibility) from $B_{e}$ to $B_{t}$ and from $B_{t}$ to $B_{t}^{*}$, respectively. In the above equations, partial differentiations are with respect to the coordinates $\tilde{x}_{i}$ in the buckled state $B_{t}$ (which is the new basic state when considering incremental displacement $\boldsymbol{v}$ ). The traction-free boundary condition $\boldsymbol{\chi} \boldsymbol{n}=0$ is applied at the image of $x_{2}=h$ in $B_{t}$, which is now curved. Similarly, traction continuity at the interface corresponds to continuity of $\boldsymbol{\chi} \boldsymbol{n}$ when evaluated at the curved interface in $B_{t}$. We also note that the instantaneous elastic moduli $\mathcal{A}_{j i l k}$, which were first defined in Chadwick and Ogden [9], depend on the deformation from $B_{e}$ to $B_{t}$ and are therefore functions of $\tilde{x}_{1}$ and $\tilde{x}_{2}$.

It is clearly more convenient to work with the counterparts of (3.1) and (3.2) expressed in terms of the coordinates $x_{i}$ (since, for instance, the free surface would 
simply correspond to $x_{2}=h$ ). The required coordinate transformation is given by (2.2). Denoting by $V$ the matrix with components $V_{i j} \equiv u_{i, j}$, we have

$$
\begin{gathered}
\frac{\partial}{\partial x_{i}}=\frac{\partial \tilde{x}_{j}}{\partial x_{i}} \frac{\partial}{\partial \tilde{x}_{j}}=\left(\delta_{i j}+\epsilon V_{j i}\right) \frac{\partial}{\partial \tilde{x}_{j}}, \\
\frac{\partial}{\partial \tilde{x}_{i}}=\left(\delta_{i j}+\epsilon Q_{i j}\right) \frac{\partial}{\partial x_{j}},
\end{gathered}
$$

where $Q_{i j}$ are the components of the cofactor matrix of $V$. In writing down the last equation, use has been made of the fact that the incremental deformation is also isochoric, so that the determinant of $I+\epsilon V$ is unity.

Expressed in terms of the coordinates $x_{i},(3.1)$ and (3.2) become

$$
\begin{gathered}
v_{i, i}+\epsilon Q_{i j} v_{i, j}=0, \quad \chi_{i j, j}+\epsilon Q_{j m} \chi_{i j, m}=0, \\
\chi_{i j}=\mathcal{A}_{j i l k}\left(v_{k, l}+\epsilon Q_{l m} v_{k, m}\right)+p\left(v_{j, i}+\epsilon Q_{i m} v_{j, m}\right)-p^{*} \delta_{j i},
\end{gathered}
$$

where here and hereafter a comma denotes differentiation with respect to the coordinates $x_{i}$ in $B_{e}$, and to simplify notation we have used the same letter to denote each function when expressed in terms of the new coordinates $x_{i}$.

We shall also need expressions for the normals to the free surface and interface in $B_{t}$. The free surface or interface has the parametric expression

$$
\tilde{x}_{1}=x_{1}+\epsilon u_{1}\left(x_{1}, x_{2}\right), \quad \tilde{x}_{2}=x_{2}+\epsilon u_{2}\left(x_{1}, x_{2}\right),
$$

where $x_{2}$ takes the value of $h$ or 0 for the free surface or interface, respectively. The normal in each case is then a multiple of $\boldsymbol{m} \equiv\left(-\epsilon u_{2,1}, 1+\epsilon u_{1,1}\right)$, and so

$$
\boldsymbol{n}=\frac{\boldsymbol{m}}{|\boldsymbol{m}|}=\left(-\epsilon u_{2,1}+\epsilon^{2} u_{2,1} u_{1,1}, 1-\frac{1}{2} \epsilon^{2} u_{2,1}^{2}\right)+O\left(\epsilon^{3}\right),
$$

which is to be evaluated at $x_{2}=h$ or 0 . Finally, the elastic moduli $\mathcal{A}_{\text {jilk }}$ are given by

$$
\mathcal{A}_{j i l k}=\left.\tilde{F}_{j A} \tilde{F}_{l B} \frac{\partial^{2} W}{\partial F_{i A} \partial F_{k B}}\right|_{F=\tilde{F}},
$$

where $F$ denotes the deformation gradient from $B_{0}$ to a general configuration and $\bar{F}$ and $\tilde{F}$ are the deformation gradients from $B_{0}$ to $B_{e}$ and from $B_{0}$ to $B_{t}$, respectively. We thus have $\tilde{F}=(I+\epsilon V) \bar{F}$. The above expression needs to be expanded around $F=\bar{F}$. In the general case, we assume that it is given by

$$
\mathcal{A}_{\text {jilk }}=\mathcal{A}_{\text {jilk }}^{(0)}+\epsilon \mathcal{A}_{\text {jilk }}^{(1)}+\epsilon^{2} \mathcal{A}_{\text {jilk }}^{(2)}+\cdots .
$$

In the case of a neo-Hookean material, we have

$$
\mathcal{A}_{j i l k}=\mu \delta_{i k} \tilde{B}_{j l}
$$

where $\mu$ is the shear modulus whose values for the layer and substrate are denoted by $\mu_{\mathrm{f}}$ and $\mu_{\mathrm{s}}$, respectively, and $\tilde{B}$ is the left Cauchy-Green strain tensor given by $\tilde{B}=\tilde{F} \tilde{F}^{T}$. From $\tilde{B}=(I+\epsilon V) \bar{B}\left(I+\epsilon V^{T}\right)$, we may obtain the asymptotic expansion of $\tilde{B}$, and hence the expansion of $\mathcal{A}_{\text {jilk }}$.

Copyright $@$ by SIAM. Unauthorized reproduction of this article is prohibited. 
TABLE 1

\begin{tabular}{|c|c|c|c|c|c|c|}
\hline$\mu_{\mathrm{f}} / \mu_{\mathrm{s}}$ & 2 & 4 & 5 & 10 & 100 & 1000 \\
\hline$\lambda_{\mathrm{cr}}$ & 0.701 & 0.810 & 0.835 & 0.895 & 0.976 & 0.995 \\
\hline$\lambda_{\mathrm{cr}}^{*}$ & 0.666 & 0.770 & 0.797 & 0.864 & 0.967 & 0.993 \\
\hline
\end{tabular}

Guided by the expansion (2.10), we now write

$$
\lambda=\lambda_{\mathrm{cr}}^{*}+\epsilon \lambda_{1}+\epsilon^{2} \lambda_{2}+\epsilon^{3} \lambda_{3}+\cdots,
$$

where $\lambda_{1}, \lambda_{2}, \lambda_{3}$ are constants to be determined and $\lambda_{\mathrm{cr}}^{*}$ is anticipated to be the critical stretch at which a mode with mode number $1 / 2$ may bifurcate from the uniformly deformed state $B_{e}$. If (3.9) is truncated at order $\epsilon^{3}$, then elimination of $\lambda$ from (2.3) and (3.9) would yield a cubic equation for $\epsilon$. Once $\epsilon$ is found, the critical value of stretch for the period-doubling secondary bifurcation is then computed from $\lambda=\lambda_{\mathrm{cr}}-\epsilon^{2}$.

In Table 1, we have listed values of $\lambda_{\mathrm{cr}}$ and $\lambda_{\mathrm{cr}}^{*}$ for a selection of ratios of the shear moduli $\mu_{\mathrm{f}}$ and $\mu_{\mathrm{s}}$. It is seen that when the ratio is greater than $100, \lambda_{\mathrm{cr}}^{*}$ is greater than 0.96 , whereas previously reported values of threshold stretch for period-doubling bifurcation are typically around 0.80 . Thus, our approach will work only if $\epsilon$ is small enough for the expansion (3.9) to be valid and at the same time large enough so that adding the $\epsilon$-dependent terms in (3.9) will produce the right reduction to bring $\lambda$ down to a value close to 0.80 . This turns out to indeed be the case.

It may be argued that (2.3) should be replaced by an expansion of the form $\lambda=\lambda_{\text {cr }}-\epsilon^{2}\left(\hat{\lambda}_{1}+\epsilon \hat{\lambda}_{2}+\epsilon^{2} \hat{\lambda}_{3}+\cdots\right)$, which is then truncated at the same order as (3.9) is truncated to ensure asymptotic consistency. However, once the calculations following this approach are completed, we can always replace $\epsilon^{2}\left(\hat{\lambda}_{1}+\epsilon \hat{\lambda}_{2}+\epsilon^{2} \hat{\lambda}_{3}+\cdots\right)$ by a new small parameter, $\hat{\epsilon}^{2}$ say, and then express $\epsilon$ asymptotically in terms of $\hat{\epsilon}$. This manipulation then recovers what is presented above.

We are now in a position to look for an asymptotic solution of the form

$$
\boldsymbol{v}=\boldsymbol{v}^{(0)}+\epsilon \boldsymbol{v}^{(1)}+\epsilon^{2} \boldsymbol{v}^{(2)}+\cdots, \quad p^{*}=\tilde{p}^{(0)}+\epsilon \tilde{p}^{(1)}+\epsilon^{2} \tilde{p}^{(2)}+\cdots .
$$

The pressure $\bar{p}$ in (2.8) depends on $\lambda$, which, because of the expansion (3.9), has the expansion

$$
\bar{p}=\bar{p}_{0}+\epsilon \bar{p}_{1}+\epsilon^{2} \bar{p}_{2}+\epsilon^{3} \bar{p}_{3}+\cdots,
$$

where all the coefficients on the right-hand side can be expressed in terms of $\lambda_{\mathrm{cr}}^{*}, \lambda_{1}, \lambda_{2}$, ... once the material model is known. For instance, when the material is neo-Hookean we have $\bar{p}=\mu \lambda^{-2}$.

On substituting these expressions into (3.5), we obtain

$$
\chi_{i j}=\chi_{i j}^{(0)}+\epsilon\left(\chi_{i j}^{(1)}+M_{i j}\right)+\epsilon^{2}\left(\chi_{i j}^{(2)}+N_{i j}\right)+\cdots,
$$

where

$$
\begin{gathered}
\chi_{i j}^{(\alpha)}=\mathcal{A}_{j i l k}^{(0)} v_{k, l}^{(\alpha)}+\bar{p}_{0} v_{j, i}^{(\alpha)}-\tilde{p}^{(\alpha)} \delta_{j i}, \quad \alpha=0,1,2, \\
M_{i j}=\mathcal{A}_{j i l k}^{(0)} Q_{l m}^{(1)} v_{k, m}^{(0)}+\mathcal{A}_{j i l k}^{(1)} v_{k, l}^{(0)}+\bar{p}_{0} Q_{i m}^{(1)} v_{j, m}^{(0)}+\left(\bar{p}_{1}+P_{1}\right) v_{j, i}^{(0)}
\end{gathered}
$$

Copyright $@$ ㅇ by SIAM. Unauthorized reproduction of this article is prohibited. 


$$
\begin{aligned}
N_{i j}= & \mathcal{A}_{j i l k}^{(0)}\left(Q_{l m}^{(2)} v_{k, m}^{(0)}+Q_{l m}^{(1)} v_{k, m}^{(1)}\right)+\mathcal{A}_{j i l k}^{(1)}\left(v_{k, l}^{(1)}+Q_{l m}^{(1)} v_{k, m}^{(0)}\right) \\
& +\mathcal{A}_{j i l k}^{(2)} v_{k, l}^{(0)}+\bar{p}_{0}\left(Q_{i m}^{(2)} v_{j, m}^{(0)}+Q_{i m}^{(1)} v_{j, m}^{(1)}\right) \\
& +\left(\bar{p}_{1}+P_{1}\right)\left(v_{j, i}^{(1)}+Q_{i m}^{(1)} v_{j, m}^{(0)}\right)+\left(\bar{p}_{2}+P_{2}\right) v_{j, i}^{(0)} .
\end{aligned}
$$

On substituting (3.10) and (3.12) into (3.4) and equating the coefficients of like powers of $\epsilon$, we obtain the following:

(1) $O\left(\epsilon^{0}\right)$ :

$$
v_{i, i}^{(0)}=0, \quad \chi_{i j, j}^{(0)}=0
$$

(2) $O\left(\epsilon^{1}\right)$ :

(3) $O\left(\epsilon^{2}\right)$ :

$$
\begin{gathered}
v_{i, i}^{(1)}=-Q_{i j}^{(1)} v_{i, j}^{(0)}, \\
\chi_{i j, j}^{(1)}=-M_{i j, j}-Q_{j m}^{(1)} \chi_{i j, m}^{(0)} .
\end{gathered}
$$

where

$$
Q_{11}^{(\alpha)}=u_{2,2}^{(\alpha)}, \quad Q_{12}^{(\alpha)}=-u_{2,1}^{(\alpha)}, \quad Q_{21}^{(\alpha)}=-u_{1,2}^{(\alpha)}, \quad Q_{22}^{(\alpha)}=u_{1,1}^{(\alpha)}, \quad \alpha=1,2 .
$$

Correspondingly, the traction vector $\boldsymbol{\chi} \boldsymbol{n}$ has the expansion

$$
\begin{aligned}
\chi_{i j} n_{j}=\chi_{i 2}^{(0)}+\epsilon\left\{\chi_{i 2}^{(1)}+M_{i 2}\right. & \left.+Q_{12}^{(1)} \chi_{i 1}^{(0)}\right\}+\epsilon^{2}\left\{\chi_{i 2}^{(2)}+N_{i 2}+Q_{12}^{(1)}\left(\chi_{i 1}^{(1)}+M_{i 1}\right)\right. \\
& +\left(u_{2,1}^{(1)} u_{1,1}^{(1)}-u_{2,1}^{(2)} \chi_{i 1}^{(0)}-\frac{1}{2} \chi_{i 2}^{(0)}\left(u_{2,1}^{(1)}\right)^{2}\right\}+O\left(\epsilon^{3}\right)
\end{aligned}
$$

so that the traction-free boundary condition at $x_{2}=h$ yields

$$
\begin{gathered}
\chi_{i 2}^{(0)}=0, \quad x_{2}=h, \\
\chi_{i 2}^{(1)}+M_{i 2}+Q_{12}^{(1)} \chi_{i 1}^{(0)}=0, \quad x_{2}=h, \\
\chi_{i 2}^{(2)}+N_{i 2}+Q_{12}^{(1)}\left(\chi_{i 1}^{(1)}+M_{i 1}\right)-\frac{1}{2} \chi_{i 2}^{(0)}\left(u_{2,1}^{(1)}\right)^{2} \\
+\left(u_{2,1}^{(1)} u_{1,1}^{(1)}-u_{2,1}^{(2)}\right) \chi_{i 1}^{(0)}=0, \quad x_{2}=h .
\end{gathered}
$$

Equations reflecting traction continuity at $x_{2}=0$ can be written down in a similar fashion. For instance, traction continuity at $x_{2}=0$ at order $\epsilon$ is given by

$$
\left[\chi_{i 2}^{(1)}+M_{i 2}+Q_{12}^{(1)} \chi_{i 1}^{(0)}\right]_{x_{2}=0}=0,
$$

where the square bracket notation is defined by

$$
[G]_{x_{2}=0}=\left.G\right|_{x_{2}=0^{+}}-\left.G\right|_{x_{2}=0^{-}} \quad \forall G,
$$

with $0^{+}$and $0^{-}$signifying approaching 0 from the positive and negative sides, respectively.

The boundary value problem at order $\epsilon^{3}$ may be obtained in a similar fashion, but it is omitted here for the sake of brevity. We note, however, that it is this problem that determines $\lambda_{3}$, and it involves the third-order term $\boldsymbol{u}^{(3)}(\boldsymbol{x})$ in the primary buckled solution (2.4).

Copyright (c) by SIAM. Unauthorized reproduction of this article is prohibited. 
4. Solution of the BVPs at different orders. We first note that the solution to the leading-order problem (3.13) subject to appropriate boundary and interface conditions is the buckling solution with mode number $1 / 2$ (since this is how $\lambda_{\mathrm{cr}}^{*}$ is defined) and can be obtained from CF. Since this is a linear problem and the amplitude is arbitrary, the solution may be in-phase or out-of-phase with the primary buckling solution, with the vertical displacement proportional to $\cos \left(x_{1} / 2\right)$ or $\sin \left(x_{1} / 2\right)$, respectively.

We now illustrate the solution of the boundary value problems (BVPs) at higher orders for the special case when the layer and substrate are each made of a neoHookean material. In this case the three governing equations at order $\epsilon^{n}$ have the forms

$$
\begin{gathered}
v_{1,1}^{(n)}+v_{2,2}^{(n)}=\sum_{a=1}^{n+1} G_{0}^{(n, a)}\left(x_{2}\right) \mathrm{e}^{\mathrm{i}\left(a-\frac{1}{2}\right) x_{1}}+\text { c.c. } \\
\mu s v_{1,11}^{(n)}+\mu s^{-1} v_{1,22}^{(n)}-p_{, 1}^{(n)}=\sum_{a=1}^{n+1} G_{1}^{(n, a)}\left(x_{2}\right) \mathrm{e}^{\mathrm{i}\left(a-\frac{1}{2}\right) x_{1}}+\text { c.c. } \\
\mu s v_{2,11}^{(n)}+\mu s^{-1} v_{2,22}^{(n)}-p_{, 2}^{(n)}=G_{2}^{(n, 0)}\left(x_{2}\right)+\sum_{a=1}^{n+1} G_{2}^{(n, a)}\left(x_{2}\right) \mathrm{e}^{\mathrm{i}\left(a-\frac{1}{2}\right) x_{1}}+\text { c.c. }
\end{gathered}
$$

where we have used $s$ to denote $\left(\lambda_{\mathrm{cr}}^{*}\right)^{2}$ to simplify notation, and $G_{\alpha}^{(n, a)}(\alpha=0,1,2$; $a=1, \ldots, n+1)$ are dependent only on solutions up to and including order $\epsilon^{n-1}$. Clearly, the solution should take the form

$$
\begin{aligned}
& \boldsymbol{v}^{(n)}=\sum_{a=1}^{n+1} \boldsymbol{V}^{(n, a)}\left(x_{2}\right) \mathrm{e}^{\mathrm{i}\left(a-\frac{1}{2}\right) x_{1}}+\text { c.c. }, \\
& p^{(n)}=\sum_{a=1}^{n+1} \Pi^{(n, a)}\left(x_{2}\right) \mathrm{e}^{\mathrm{i}\left(a-\frac{1}{2}\right) x_{1}}+\text { c.c. }
\end{aligned}
$$

where the unknown functions $\boldsymbol{V}^{(n, a)}$ and $\Pi^{(n, a)}$ are determined by solving the reduced equations

$$
\begin{gathered}
\mathrm{i} \hat{a} V_{1}^{(n, a)}\left(x_{2}\right)+V_{2}^{(n, a)^{\prime}}\left(x_{2}\right)=G_{0}^{(n, a)}\left(x_{2}\right), \\
-\hat{a}^{2} \mu s V_{1}^{(n, a)}\left(x_{2}\right)+\mu s^{-1} V_{1}^{(n, a)^{\prime}}\left(x_{2}\right)-\mathrm{i} \hat{a} \Pi^{(n, a)}\left(x_{2}\right)=G_{1}^{(n, a)}\left(x_{2}\right), \\
-\hat{a}^{2} \mu s V_{2}^{(n, a)}\left(x_{2}\right)+\mu s^{-1} V_{2}^{(n, a)^{\prime}}\left(x_{2}\right)-\Pi^{(n, a)^{\prime}}\left(x_{2}\right)=G_{2}^{(n, a)}\left(x_{2}\right) .
\end{gathered}
$$

In the above equations, we have used $\hat{a}$ to denote $a-1 / 2$, and a prime denotes differentiation with respect to $x_{2}$. Closed-form solutions for the above equations can be found as follows.

First, we note that (4.6) and (4.7) can be used to express $V_{1}^{(n, a)}$ and $\Pi^{(n, a)}$ in terms of $V_{2}^{(n, a)}$. Equation (4.8) can then be reduced to the form

$$
V_{2}^{(n, a)^{\prime \prime \prime \prime}}-\left(1+s^{2}\right) \hat{a}^{2} V_{2}^{(n, a)^{\prime \prime}}+s^{2} \hat{a}^{4} V_{2}^{(n, a)}=G_{3}^{(n, a)}\left(x_{2}\right),
$$

Copyright $@$ by SIAM. Unauthorized reproduction of this article is prohibited. 
where $G_{3}^{(n, a)}\left(x_{2}\right)$ is a known function at this stage of the analysis. The general solution is then given by

$$
V_{2}^{(n, a)}\left(x_{2}\right)=c_{1} \mathrm{e}^{\hat{a} x_{2}}+c_{2} \mathrm{e}^{\hat{a} s x_{2}}+c_{3} \mathrm{e}^{-\hat{a} x_{2}}+c_{4} \mathrm{e}^{-\hat{a} s x_{2}}+\text { P.I. },
$$

where $c_{1}, c_{2}, c_{3}, c_{4}$ are constants and P.I. stands for a particular integral. When the above form is specialized to the substrate, the constants $c_{3}$ and $c_{4}$ need to be set to zero in order to satisfy the decaying condition.

For the substrate a particular integral can be obtained by using Fourier transforms or the Laplace transforms method. It is straightforward to show that a particular integral is given by

$$
\begin{aligned}
\text { P.I. }=\frac{1}{2 \hat{a}^{3} s\left(1-s^{2}\right)} & \left\{s \mathrm{e}^{\hat{a} x_{2}} \int_{0}^{x_{2}} \mathrm{e}^{-\hat{a} t} G_{3}^{(n, a)}(t) d t-\mathrm{e}^{\hat{a} s x_{2}} \int_{0}^{x_{2}} \mathrm{e}^{-\hat{a} s t} G_{3}^{(n, a)}(t) d t\right. \\
+ & \left.\mathrm{e}^{-\hat{a} s x_{2}} \int_{-\infty}^{x_{2}} \mathrm{e}^{-\hat{a} s t} G_{3}^{(n, a)}(t) d t-s \mathrm{e}^{-\hat{a} x_{2}} \int_{-\infty}^{x_{2}} \mathrm{e}^{\hat{a} t} G_{3}^{(n, a)}(t) d t\right\} .
\end{aligned}
$$

For the layer, neither Fourier transforms nor the Laplace transforms method can be applied directly. Instead, the method of variation of parameters can be applied. More simply, a particular integral can be obtained by replacing the lower limit $-\infty$ by 0 in the above expression. It has been verified that the resulting expression is the same as that obtained by using the method of variation of parameters.

We thus have two expressions for $V_{2}^{(n, a)}$ valid for the layer and substrate, respectively. On substituting these expressions into the associated boundary and interface conditions, we would obtain a matrix equation of the form $M \boldsymbol{c}=\boldsymbol{b}$, where $\boldsymbol{c}$ is the 6 -vector formed from the four constants for the layer and two constants for the substrate. When $a \neq 1$, the coefficient matrix $M$ is invertible, and a unique solution for $c$ can be found. For $a=1$, the coefficient matrix $M$ is singular since $\lambda_{\mathrm{cr}}^{*}$ satisfies $\operatorname{det} M=0$. A solvability condition needs to be imposed on $\boldsymbol{b}$, and this condition then determines the $\lambda_{n}$ in (3.9).

We note that this solution procedure may also be applied to the primary postbuckling problem discussed in section 2 when $\lambda_{\mathrm{cr}}^{*}$ is replaced by $\lambda_{\mathrm{cr}}$.

We have carried out numerical experiments to verify that the general solution of (4.9) can also be obtained by using the command DSolve on Mathematica directly, provided that a high-enough working precision is set. For the purpose of determining the first four terms in the expansion (3.9), it is sufficient to set the working precision to be 180 if the above closed-form solutions are used, and to be 280 if the command DSolve is used to find the general solution of (4.9). Thus, although when more general material models are used, the above closed-form solutions may not be possible, the required general solution can still be obtained numerically with the aid of Mathematica.

5. Alternative method for finding $\boldsymbol{\lambda}_{\mathbf{3}}$. If the expansion (3.9) is truncated at order $\epsilon^{3}$, then the last term $\lambda_{3}$ can be determined by using the following alternative procedure that does not require the solution of the BVP for $V_{2}^{(3,1)}\left(x_{2}\right)$ explicitly. This can reduce the computing time significantly, especially when equations such as (4.9) are solved numerically as discussed above. The same procedure can also be used to determine $\lambda_{1}$ and $\lambda_{2}$ as useful checks.

To determine $\lambda_{n}$, where $n=1,2$, or 3 , we consider the contour integral $I$ defined by

$$
I=\oint_{\partial S_{c}} v_{i}^{(0)} \chi_{i j}^{(n)} n_{j} d s+\oint_{\partial S_{b}} v_{i}^{(0)} \chi_{i j}^{(n)} n_{j} d s, \quad n=1,2,3
$$

Copyright (c) by SIAM. Unauthorized reproduction of this article is prohibited. 
where the domains $S_{c}$ and $S_{b}$ are defined by

$$
\begin{gathered}
S_{c}: 0 \leq x_{1} \leq 4 \pi, \quad 0 \leq x_{2} \leq h, \\
S_{b}: 0 \leq x_{1} \leq 4 \pi, \quad-L \leq x_{2} \leq 0,
\end{gathered}
$$

respectively. In the above definition, $L$ is a sufficiently large positive constant. In each integral, the integrand is $4 \pi$-periodic, and so the contributions from the two vertical sides $x_{1}=0,4 \pi$ cancel each other. With the additional use of the fact that the integrand tends to zero as $x_{2} \rightarrow-\infty$, we obtain

$$
\lim _{L \rightarrow \infty} I=\left.\int_{0}^{4 \pi} v_{i}^{(0)} \chi_{i 2}^{(n)}\right|_{x_{2}=h} d x_{1}-\int_{0}^{4 \pi} v_{i}^{(0)}\left[\chi_{i 2}^{(n)}\right]_{x_{2}=0} d x_{1},
$$

where the bracket notation is defined by (3.24).

On the other hand, with the use of the divergence theorem, we may also write

$$
\begin{aligned}
I & =\int_{-L}^{h} \int_{0}^{4 \pi}\left(v_{i, j}^{(0)} \chi_{i j}^{(n)}+v_{i}^{(0)} \chi_{i j, j}^{(n)}\right) d x_{1} d x_{2} \\
& =\int_{-L}^{h} \int_{0}^{4 \pi}\left(v_{i, j}^{(n)} \chi_{i j}^{(0)}+\tilde{p}^{(0)} v_{i, i}^{(n)}+v_{i}^{(0)} \chi_{i j, j}^{(n)}\right) d x_{1} d x_{2},
\end{aligned}
$$

where in writing down the last step use has been made of the pairwise symmetry property $\mathcal{A}_{j i l k}^{(0)}=\mathcal{A}_{l k j i}^{(0)}$. By integration by parts, followed by the use of the leading-order equations (3.13) and associated boundary condition (3.20), the integral of $v_{i, j}^{(n)} \chi_{i j}^{(0)}$ can be shown to be zero, and so it follows that

$$
I=\int_{-L}^{h} \int_{0}^{4 \pi}\left(\tilde{p}^{(0)} v_{i, i}^{(n)}+v_{i}^{(0)} \chi_{i j, j}^{(n)}\right) d x_{1} d x_{2} .
$$

On substituting this expression back into (5.2), we obtain

$$
\begin{aligned}
\int_{-\infty}^{h} \int_{0}^{4 \pi}\left(\tilde{p}^{(0)} v_{i, i}^{(n)}+v_{i}^{(0)} \chi_{i j, j}^{(n)}\right) d x_{1} d x_{2} \\
\quad=\int_{0}^{4 \pi}\left(v_{i}^{(0)} \chi_{i 2}^{(n)}\right)_{x_{2}=h} d x_{1}-\int_{0}^{4 \pi} v_{i}^{(0)}\left[\chi_{i 2}^{(n)}\right]_{x_{2}=0} d x_{1}, \quad n=1,2 .
\end{aligned}
$$

These are the equations that can be used to find $\lambda_{n}(n=1,2,3)$. For instance, when $n$ is equal to 1 or 2 , the $v_{i, i}^{(1)}, \chi_{i j, j}^{(1)}$, and $\chi_{i 2}^{(1)}$ at $x_{2}=h$ can be eliminated with the aid of (3.14), (3.15), and (3.21), respectively, whereas the factors $v_{i, i}^{(2)}, \chi_{i j, j}^{(2)}$, and $\chi_{i 2}^{(2)}$ at $x_{2}=h$ can be eliminated with the aid of (3.16), (3.17), and (3.22), respectively. The jumps $\left[\chi_{i 2}^{(1)}\right]$ and $\left[\chi_{i 2}^{(2)}\right]$ at $x_{2}=0$ can each be expressed in terms of solutions at one order lower with the aid of the traction continuity condition $\left[\chi_{i 2}\right]=0$ at $x_{2}=0$ together with the expansion (3.19). For instance, the jump $\left[\chi_{i 2}^{(1)}\right]$ can be obtained from (3.23). Finally, in evaluating the above expressions, only those terms that are independent of $x_{1}$ will survive the integration. This virtual work method is underlined by the same principle as obtaining the solvability condition $(2.15)_{1}$ by contracting (2.13) with $\sin \left(x_{1} / 2\right)$ and then integrating the resulting equation from 0 to $4 \pi$. It avoids having to solve the BVP for which a solvability condition needs to be imposed. 
TABLE 2

\begin{tabular}{|c|c|c|c|c|c|c|c|}
\hline$\mu_{\mathrm{f}} / \mu_{\mathrm{s}}$ & $\lambda_{\mathrm{cr}}$ & $\lambda_{\mathrm{cr}}^{*}$ & $\lambda_{1}$ & $\lambda_{2}$ & $\lambda_{3}$ & $\lambda_{\text {sec }}$ & $\epsilon$ \\
\hline 5 & 0.835 & 0.797 & 0.112 & -1.39 & 0.0162 & -558.3 & 23.6 \\
5.5 & 0.845 & 0.808 & 0.0999 & -1.32 & 0.183 & -1.279 & 1.46 \\
5.75 & 0.850 & 0.812 & 0.0945 & -1.29 & 0.237 & -0.1088 & 0.979 \\
5.799 & 0.850 & 0.813 & 0.0935 & -1.28 & 0.246 & -0.001361 & 0.923 \\
5.8 & 0.850 & 0.813 & 0.0935 & -1.28 & 0.246 & 0.0006128 & 0.922 \\
6 & 0.854 & 0.817 & 0.0897 & -1.27 & 0.277 & 0.2760 & 0.760 \\
6.5 & 0.861 & 0.825 & 0.0812 & -1.22 & 0.331 & 0.5257 & 0.579 \\
7 & 0.868 & 0.833 & 0.0741 & -1.19 & 0.361 & 0.6096 & 0.508 \\
8 & 0.879 & 0.845 & 0.0627 & -1.15 & 0.383 & 0.6753 & 0.451 \\
10 & 0.895 & 0.864 & 0.0473 & -1.09 & 0.371 & 0.7197 & 0.418 \\
30 & 0.948 & 0.930 & 0.0115 & -1.003 & 0.181 & 0.7672 & 0.425 \\
120 & 0.979 & 0.971 & 0.00183 & -0.995 & 0.0662 & 0.7717 & 0.455 \\
448 & 0.991 & 0.988 & 0.000315 & -0.997 & 0.0266 & 0.7706 & 0.470 \\
600 & 0.993 & 0.990 & 0.000213 & -0.997 & 0.0218 & 0.7703 & 0.471 \\
774 & 0.994 & 0.991 & 0.000151 & -0.998 & 0.0183 & 0.7701 & 0.473 \\
\hline
\end{tabular}

In our illustrative calculations for neo-Hookean materials, the above approach is also applied to the reduced problem (4.9) as a useful check. In this case the counterpart of (5.5) is obtained by contracting (4.9) corresponding to $a=0$ with $V_{2}^{(0,0)}$, and then integrating the resulting expression from $-\infty$ to $h$. The function $V_{2}^{(n, 0)}\left(x_{2}\right)$ can be eliminated by integration by parts, followed by the use of the associated boundary and interface conditions. Thus, to find $\lambda_{3}$ we do not need to determine $V_{2}^{(3,0)}\left(x_{2}\right)$ explicitly; only the lower-order solutions are required.

6. Numerical results. As remarked earlier, on truncating the expansion (3.9) at order $\epsilon^{3}$ and eliminating $\lambda$ from (2.3) and (3.9), we obtain a cubic equation for $\epsilon$ :

$$
\lambda_{3} \epsilon^{3}+\left(1+\lambda_{2}\right) \epsilon^{2}+\lambda_{1} \epsilon+\lambda_{\mathrm{cr}}^{*}-\lambda_{\mathrm{cr}}=0 .
$$

This equation is found to have a unique real and positive root for all values of $\mu_{\mathrm{f}} / \mu_{\mathrm{s}}$ considered in this paper. Once $\epsilon$ is found in this way, the associated threshold value of $\lambda$, say $\lambda_{\mathrm{sec}}$, is computed from $\lambda_{\mathrm{sec}}=\lambda_{\mathrm{cr}}-\epsilon^{2}$. In Table 2 we have listed the coefficients in (6.1) and the predicted values of $\lambda_{\sec }$ for different values of the modulus ratio. The given results correspond to bifurcation modes that are out-of-phase with the primary buckling mode. The solutions corresponding to bifurcation modes that are in-phase with the primary buckling mode are the same except that the signs of $\lambda_{1}, \lambda_{3}$, and hence $\epsilon$, are changed. However, changing the sign of $\epsilon$ is equivalent to replacing $x_{1}$ by $x_{1}+\pi$ in the primary buckling solution, and the latter shift in $x_{1}$ would make the period-doubling perturbation out-of-phase again. Thus, we may assume $\epsilon$ to be positive without loss of generality, and we conclude that the period-doubling solution is unique.

For the special case when both the layer and substrate are neo-Hookean, fully numerical simulations have been carried out by Cao and Hutchinson [8], and more recently by Zhao et al. [17]. Our asymptotic results show excellent agreement with their numerical results in a number of ways. First, for the three cases of $\mu_{\mathrm{f}} / \mu_{\mathrm{s}}=30,448$, and 774 , their numerical values for $\lambda_{\text {sec }}$ are equal to $0.8,0.815,0.815$, respectively. Our asymptotic results in Table 2 approximate these values with a relative error equal to $4.1 \%, 5.5 \%$, and $5.5 \%$, respectively. Second, both their numerical simulations and our asymptotic results show the trend that for modulus ratios larger than 100 the value of $\lambda_{\mathrm{sec}}$ becomes insensitive to further increases of this modulus ratio. In other words, 
in the limit $\mu_{\mathrm{f}} / \mu_{\mathrm{s}} \rightarrow \infty$, the value of $\lambda_{\mathrm{sec}}$ tends to an asymptotic limit rapidly. Third, our asymptotic predictions for $\lambda_{\mathrm{sec}}$ are always smaller than their numerical simulation counterparts. This is consistent with the expectation that the critical bifurcation strain (which is $1-\lambda_{\mathrm{sec}}$ ) in the presence of imperfections should be smaller than the corresponding value associated with a perfect system. Some sort of imperfection was always introduced in numerical simulations, whereas our asymptotic analysis was conducted for a perfect bilayer system.

We may also compare our asymptotic result with the set of experimental results corresponding to system 2 in Brau et al. [4]. It follows from the given ratio of Young's modulus that $\mu_{\mathrm{f}} / \mu_{\mathrm{s}}=120$, and it seems from the caption of their Figure 1 that period-doubling occurred when the stretch reached the value of 0.81 . Our predicted value of 0.77 in Table 2 then only differs from the given experimental value with a relative error of $4.9 \%$.

Our asymptotic results in Table 2 also predict that period-doubling bifurcation becomes impossible when the modulus ratio becomes less than approximately 5.8 . However, since our asymptotic results become invalid in this regime, we expect the value 5.8 to be only a crude estimate. There do not seem to exist any numerical or experimental results confirming this qualitative prediction, but there is a consensus in the literature that period-doubling is possible only when the modulus ratio is large enough, although the minimum modulus ratio for this to occur has previously not been given. We note, however, that when the modulus ratio becomes less than 1.74, the primary bifurcation becomes subcritical and so period-doubling is definitely not possible; see CF or Hutchinson [11].

7. Conclusion. In this paper we have derived an asymptotic expression for the threshold value of the principal stretch at which a secondary period-doubling bifurcation occurs in a uni-axially compressed film-substrate bilayer structure. Our calculation is based on the exact theory of nonlinear elasticity, and the approach is selfconsistent. For each small but fixed value of $\epsilon$, with the associated principal stretch given by $\lambda=\lambda_{\mathrm{cr}}-\epsilon^{2}$, the primary buckling solution is determined up to and including order $\epsilon^{3}$ and consists of Fourier modes up to and including $\cos 3 x_{1}$. Since it is well recognized that asymptotic expansions usually provide very good approximations even when the small parameter is only moderately small, and Fourier expansions usually converge fast when the functions to be approximated are periodic and smooth, we can safely expect that the weakly nonlinear postbuckling solution provides a very good approximation for the exact solution when $\epsilon$ is less than 0.5 ; see the last column in Table 2. Once this primary buckling solution can be determined for each $\epsilon$, we increase $\epsilon$ gradually and determine its threshold value at which the primary buckled configuration suffers a secondary bifurcation in which the buckling mode doubles the period of the original buckling mode. This secondary bifurcation problem is solved by using the well-established incremental theory of nonlinear elasticity and treating the secondary period-doubling bifurcation as a subharmonic resonance phenomenon. In solving the incremental problem, the principal stretch is expanded as $\lambda=\lambda_{\text {cr }}^{*}+$ $\epsilon \lambda_{1}+\epsilon^{2} \lambda_{2}+\epsilon^{3} \lambda_{3}+\cdots$, and the coefficients $\lambda_{1}, \lambda_{2}$, and $\lambda_{3}$ are determined from three solvability conditions imposed at three consecutive orders.

With excellent agreement with the fully numerical simulation results achieved, we may make a few additional observations. First, if only Fourier modes up to and including $\cos 2 x_{1}$ are included in the primary deformation, as was done in some previous studies, then $\lambda$ can only be determined self-consistently up to and including order 
$\epsilon^{2}$. In this case the threshold value of $\epsilon$ is determined from the quadratic equation

$$
\left(1+\lambda_{2}\right) \epsilon^{2}+\lambda_{1} \epsilon+\lambda_{\mathrm{cr}}^{*}-\lambda_{\mathrm{cr}}=0,
$$

which either does not have a real solution at all (if $1+\lambda_{2}<0$ ) or has a positive solution which yields a negative value for $\lambda_{\mathrm{sec}}$. Thus, what has been carried out in this paper is the minimum order of expansions that can give a realistic prediction for $\lambda_{\mathrm{sec}}$.

In order to see how including higher-order terms affects our predictions, we have also computed the next two terms in the expansion (3.9) for the three cases of $\mu_{\mathrm{f}} / \mu_{\mathrm{s}}=$ 30,448 , or 774 . To this end, the primary buckled state is determined up to and including order $\epsilon^{5}$ (and the $O\left(\epsilon^{5}\right)$ solution is determined completely by a solvability condition at $\left.O\left(\epsilon^{7}\right)\right)$. It is found that

$$
\begin{gathered}
\lambda_{4}=-0.169148, \quad \lambda_{5}=0.0354227 \quad \text { when } \mu_{\mathrm{f}} / \mu_{\mathrm{s}}=30, \\
\lambda_{4}=-0.00002060, \quad \lambda_{5}=-0.00286524 \quad \text { when } \mu_{\mathrm{f}} / \mu_{\mathrm{s}}=448,
\end{gathered}
$$

and

$$
\lambda_{4}=0.00141285, \quad \lambda_{5}=-0.00429210 \quad \text { when } \mu_{\mathrm{f}} / \mu_{\mathrm{s}}=774
$$

Denoting the threshold values of $\epsilon$ and $\lambda$ by $\epsilon^{(n)}$ and $\lambda_{\text {sec }}^{(n)}$, respectively, when the expansion (3.9) is truncated at order $\epsilon^{n}$, we have

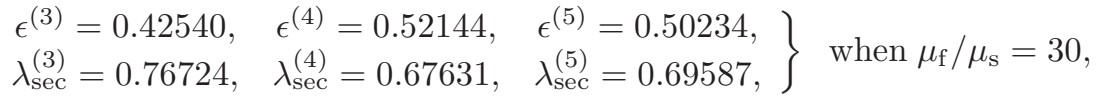

$$
\begin{aligned}
& \left.\begin{array}{lll}
\epsilon^{(3)}=0.46969, & \epsilon^{(4)}=0.46974, & \epsilon^{(5)}=0.47299, \\
\lambda_{\text {sec }}^{(3)}=0.77058, & \lambda_{\text {sec }}^{(3)}=0.77054, & \lambda_{\text {sec }}^{(5)}=0.76747,
\end{array}\right\} \quad \text { when } \mu_{\mathrm{f}} / \mu_{\mathrm{s}}=448,
\end{aligned}
$$

and

$$
\left.\begin{array}{lll}
\epsilon^{(3)}=0.47308, & \epsilon^{(4)}=0.46835, & \epsilon^{(5)}=0.47527, \\
\lambda_{\mathrm{sec}}^{(3)}=0.77007, & \lambda_{\mathrm{sec}}^{(4)}=0.77451, & \lambda_{\mathrm{sec}}^{(5)}=0.76799,
\end{array}\right\} \quad \text { when } \mu_{\mathrm{f}} / \mu_{\mathrm{s}}=774
$$

It is seen that when higher-order terms in (3.9) are included, the change in $\lambda_{\sec }$ is negligible when the modulus ratio is high but seems to be more appreciable when the modulus ratio becomes smaller.

To conclude, this first study on period-doubling using the exact theory of nonlinear elasticity has focused on the feasibility of the methodology proposed. It would seem that this methodology could be used to study a variety of secondary bifurcation problems. In particular, for the film/substrate bilayer structure which features in a variety of applications, it is of interest to investigate systematically how period-doubling is affected by compressibility, a prestretch in the substrate, and use of different material models. This will be carried out in a separate study.

Acknowledgment. The authors wish to thank Professor Yanping Cao of Tsinghua University for drawing their attention to the problem of period-doubling in film-substrate bilayers. 


\section{REFERENCES}

[1] B. Audoly And A. Boudaoud, Buckling of a stiff film bound to a compliant substrate-Part I: Formulation, linear stability of cylindrical patterns, secondary bifurcations, J. Mech. Phys. Solids, 56 (2008), pp. 2401-2421.

[2] N. Bowden, S. Brittain, A. G. Evans, J. W. Hutchinson, and G. M. Whitesides, Spontaneous formation of ordered structures in thin films of metals supported on an elastomeric polymer, Nature, 393 (1998), pp. 146-149.

[3] N. Bowden, W. T. S. Huck, K. E. Paul, And G. M. Whitesides, The controlled formation of ordered, sinusoidal structures by plasma oxidation of an elastomeric polymer, Appl. Phys. Lett., 75 (1999), pp. 2557-2559.

[4] F. Brau, H. Vandeparre, A. Sabbah, C. Poulard, A. Boudaoud, and P. Damman, Multiple-length-scale elastic instability mimics parametric resonance of nonlinear oscillators, Nat. Phys., 7 (2011), pp. 56-60.

[5] S. Budday, E. Kuhl, And J. W. Hutchinson, Period-doubling and period-tripling in growing bilayered systems, Phil. Mag., (2015), doi:10.1080/14786435.2015.1014443.

[6] Z. X. CAi And Y. B. Fu, On the imperfection sensitivity of a coated elastic half-space, Proc. R. Soc. Lond. A, 455 (1999), pp. 3285-3309.

[7] Z. X. CAI AND Y. B. FU, Exact and asymptotic stability analyses of a coated elastic half-space, Internat. J. Solids Structures, 37 (2000), pp. 3101-3119.

[8] Y. P. CAO AND J. W. Hutchinson, Wrinkling phenomena in neo-Hookean film/substrate bilayers, ASME J. Appl. Mech., 79 (2012), 031019.

[9] P. Chadwick and R. W. Ogden, On the definition of elastic moduli, Arch. Ration. Mech. Anal., 44 (1971), pp. 41-53.

[10] Y. B. Fu And R. W. Ogden, Nonlinear stability analysis of pre-stressed elastic bodies, Contin. Mech. Thermodyn., 11 (1999), pp. 141-172.

[11] J. W. Hutchinson, The role of nonlinear substrate elasticity in the wrinkling of thin films, Phil. Trans. R. Soc. A, 371 (2013), 20120422.

[12] B. Li, Y. P. CaO, X. Q. Feng, and H. J. GaO, Mechanics of morphological instabilities and surface wrinkling in soft materials: A review, Soft Matter, 8 (2012), pp. 5728-5745.

[13] A. H. Nayfeh and D. T. Mook, Nonlinear Oscillations, Wiley, New York, 1995.

[14] R. W. Ogden, Non-Linear Elastic Deformations, Ellis Horwood, New York, 1984.

[15] L. Pocivavsek, R. Dellsy, A. Kern, S. Johnson, B. H. Lin, K. Y. C. Lee, and E. Cerda, Stress and fold localization in thin elastic membranes, Science, 320 (2008), pp. 912-916.

[16] J. Y. Sun, S. Xia, M. Y. Moon, K. H. OH, And K. S. Kim, Folding wrinkles of a thin stiff layer on a soft substrate, Proc. R. Soc. Lond. A, 468 (2011), pp. 932-953.

[17] Y. Zhao, Y. P. Cao, W. Hong, M. K. Wadee, and X. Q. Feng, Towards a quantitative understanding of period-doubling wrinkling patterns occurring in film/substrate bilayer systems, Proc. Roy. Soc. Lond. A, 471 (2015), 20140695.

[18] L. ZhUO AND Y. Zhang, The mode-coupling of a stiff film/compliant substrate system in the post-buckling range, Internat. J. Solids Structures, 53 (2015), pp. 28-37.

[19] S. Wolfram, Mathematica: A System for Doing Mathematics by Computer, Addison-Wesley, San Francisco, 1991.

Copyright (c) by SIAM. Unauthorized reproduction of this article is prohibited. 